\title{
Complete genome sequence of the novel duck hepatitis B virus strain SCP01 from Sichuan Cherry Valley duck
}

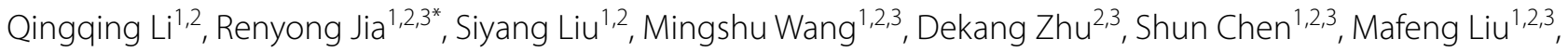
Zhongqiong Yin ${ }^{3}$, Bo Jing ${ }^{3}$ and Anchun Cheng ${ }^{1,2,3^{*}}$

\begin{abstract}
Background: The duck hepatitis B virus (DHBV) strain, designated SCP01, was isolated and identified from a Sichuan Cherry Valley duck in Southwestern China. To determine the origination and evolution of this isolated strain, we carried out complete genome sequencing of this strain.

Findings: Sequencing of the nucleotide sequence of DHBV strain SCP01 revealed a genome size of 3021 bp that contained three open reading frames, designated as $C, S$, and $P$, which were consistent with those of other duck hepatitis B viruses nucleotide sequences available in the GenBank of NCBI. Sequence comparisons based on the full-length genomic sequences showed that the DHBV SCP01 strain had the highest similarity (99.64\%) with the sequence of strain DHBV-XY, but had a lower similarity (90.04\%) with the sequence of strain DHBV CH5 isolated from Southwestern China. Phylogenetic analysis revealed that the DHBV-XY and DHBV SCP01 formed a branch that was clearly distinct from the other strains.

Conclusion: This study show that the DHBV SCP01 strain from Sichuan belonged to "Western" isolates, while the DHBV CH5 from Sichuan belonged to "Chinese" isolates. These data will promote further research into the evolutionary biology, epidemiology and pathobiology of hepadnavirus infections. In addition, continuing duck hepatitis B virus surveillance in poultry is critical to understand the patterns of DHBV infection, and to find further animal infection models to study HBV infection.
\end{abstract}

Keywords: Duck hepatitis B virus, Cherry valley duck, Phylogenetic analysis, Complete genome sequence, Chinese and Western isolates

\section{Background}

Duck hepatitis B virus (DHBV), first discovered in Peking ducks in 1980 (Mason et al. 1980), and subsequently reported in Germany and other countries throughout the world (Mattes et al. 1990; Triyatni et al. 2001; Mangisa et al. 2004; Liu et al. 2014), is a member of the genus Avihepadnavirus, family Hepadnaviridae. The genome of DHBV, a complete minus and incomplete plus strand, is circular and approximately $3.0 \mathrm{~kb}$ in length (Cova et al. 2011). The genomic DNA is maintained in a circular

\footnotetext{
*Correspondence: jiary@sicau.edu.cn; chenganchun@vip.163.com

${ }^{1}$ Avian Disease Research Center, Sichuan Agricultural University, Chengdu 611130, China

Full list of author information is available at the end of the article
}

conformation by a short cohesive overlap between the two DNA strands (Molnar-Kimber et al. 1984). DHBV is similar to hepatitis B virus (HBV) in terms of genetic organization and virus replication, and causes speciesspecific transient (acute) or persistent infection (Jilbert and Kotlarski 2000). There are approximately 240 million people worldwide that are chronically infected with HBV (Lavanchy and Kane 2016). Chronic HBV infection results in liver disease, including inflammation, fibrosis, cirrhosis and hepatocellular carcinoma (HCC) (Feng et al. 2010). DHBV does not lead to severe clinical disease in ducks or a drop in productivity, but serves as an animal infection model of human HBV, and has been used widely for comparative studies. 


\section{Methods}

Virus isolation

Positive serum screened by PCR using the primers P1 and P2 (Table 1) was collected and filtered through a $0.22 \mu \mathrm{m}$ filter. The 9-day-old duck embryonated eggs were inoculated with the filtered suspension $(100 \mu \mathrm{L} / \mathrm{embryo})$ into the allantoic cavity and then cultured in a $37{ }^{\circ} \mathrm{C}$ incubator and checked daily. The allantoic fluid was harvested at 4 days after inoculation and then for another round of inoculation.

\section{Nucleotide sequencing of the complete genome}

Viral DNA was extracted from $100 \mu \mathrm{L}$ of allantoic fluid using the TIANamp Virus DNA/RNA Kit (TIANGEN BIOTECH, Beijing, China) according to the manufacturer's instructions. Afterwards, based on the multiple alignments of the complete genome of DHBV available in GenBank, two pairs of primers were designed using Primer Premier 5 software (Table 1; Fig. 1) (Günther et al. 1995). Primers P3 and P4 were designed to amplify the complete genome sequence of DHBV, the PCR amplification was performed in a $50 \mu \mathrm{L}$ mixture containing $0.5 \mu \mathrm{L} \mathrm{LAmp}{ }^{\mathrm{TM}}$ DNA polymerase $(5 \mathrm{U} / \mu \mathrm{L}), 1 \mu \mathrm{L}$ dNTP Mix (10 Mm each), $5 \mu \mathrm{L} 10 \times$ LAmp $^{\text {TM }}$ buffer (with $20 \mathrm{mM} \mathrm{MgCl}_{2}$ ), $1 \mu \mathrm{L}$ viral DNA template, $2 \mu \mathrm{L}$ each of primers P3 and P4 $(10 \mu \mathrm{M})$, and $38.5 \mu \mathrm{L} d d \mathrm{H}_{2} \mathrm{O}$. The amplification procedure consisted of denaturation at $94{ }^{\circ} \mathrm{C}$ for $5 \mathrm{~min}$ followed by 32 cycles of denaturation at $94{ }^{\circ} \mathrm{C}$ for $30 \mathrm{~s}$, annealing at $57{ }^{\circ} \mathrm{C}$ for $30 \mathrm{~s}$, extension at $72{ }^{\circ} \mathrm{C}$ for $3 \mathrm{~min} 10 \mathrm{~s}$, and then a final extension at $72{ }^{\circ} \mathrm{C}$ for $7 \mathrm{~min}$. Primers P5 and P6 were used to amplify the region including the sequences of $\mathrm{P} 3$ and $\mathrm{P} 4$, which PCR conditions were similar to the above amplification. The amplified products were purified using the Universal DNA Purification Kit and cloned into the pGM-T vector (TIANGEN BIOTECH), and then sequenced using classical dideoxy Sanger sequencing (TSINGKE, Chengdu, China). The sequences were assembled using the Chromas software package (http://www.Technelysium.Com.au/chromas.html) to produce the final genome sequence.

\section{Table 1 Primers for PCR amplification}

\begin{tabular}{ll}
\hline Name & Sequence $\left(\mathbf{5}^{\prime} \mathbf{-} \mathbf{3}^{\prime}\right)$ \\
\hline P1 & ATGTCTGGTACCTTCGGGGGA \\
P2 & CTAACTCTTGTAAAAAAGAGC \\
P3 & AATTACACCCCTCTCCTTCGGAG \\
P4 & GTAATTCTTAAGTTCCACATAGCC \\
P5 & CACCCCTCTCTCGAAAGCAATA \\
P6 & GATAGTCAGGTTGAAAGCTCAC \\
\hline
\end{tabular}

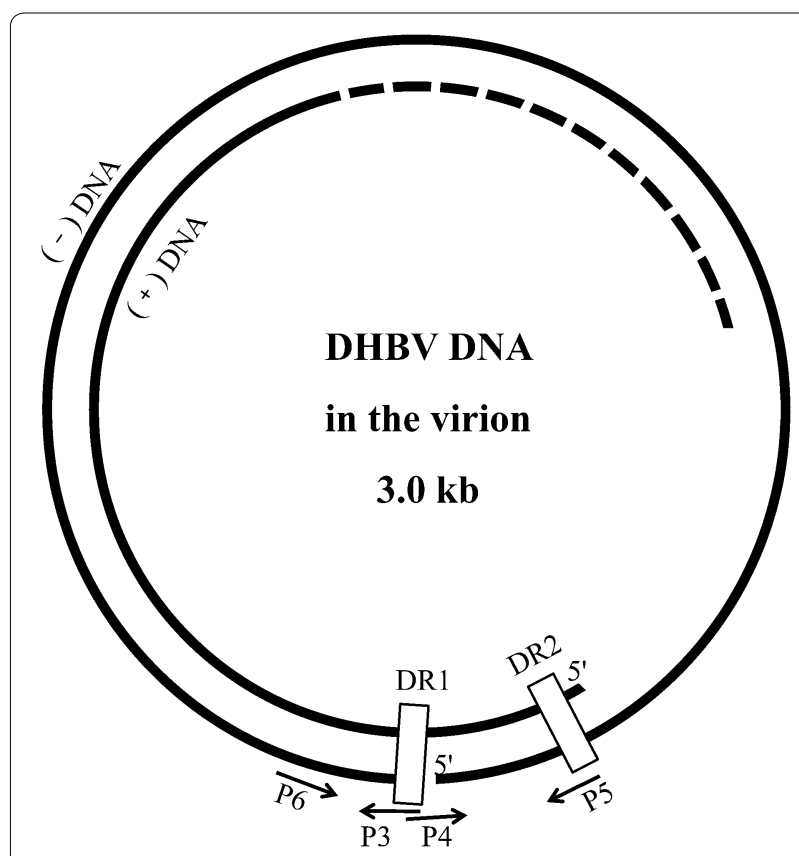

Fig. 1 Schematic representation and primers used for amplification of the whole DHBV genomes. The whole DHBV genomes are open circular DHBV virion DNA, which contain a complete minus and incomplete plus strand. The genomic DNA is maintained in a circular conformation by a short cohesive overlap between the two DNA strands, the cohesive overlap region is located between DR1 and DR2. The triple-strand region contains the terminal redundany of the minus strand, regions of the viral genome containing DR1 and DR2 correspond to the sites of initiation of synthesis of the viral minus and plus strands. Primer P3 and P4 is used to amplify the complete genome sequence of DHBV in region of DR1, primer P5 and P6 is designed to amplify the sequence including the region of primer P3 and $\mathrm{P} 4$ for ensuring the full gene sequence correctly. $D R$ direct repeat

\section{Genetic characterization}

To exhibit the genome features of the SCP01 strain, sequence analysis was conducted using the DNAMAN program. The open reading frames (ORFs) were identified according to the online tool ORF Finder (http://www. ncbi.nlm.nih.gov/gorf/gorf.html) in National Center for Biotechnology Information (NCBI).

\section{Phylogenetic analysis}

Phylogenetic analyses were performed using the maximum likelihood method with the use of Mega5.1 software (Tamura et al. 2011). Initial trees for the heuristic search were obtained by applying the neighbor-joining method to a matrix of pairwise distances estimated using the maximum composite likelihood approach. The bootstrap consensus trees inferred from 1000 replicates and branches corresponding to partitions reproduced in less than $70 \%$ bootstrap replicates were collapsed. 


\section{Results and discussion}

In June 2014, DHBV strain SCP01 was isolated from ducklings in a commercial Cherry Valley duck breeding company in Sichuan Province, Southwestern China. Subsequently, the nucleotide sequence of DHBV strain SCP01 was amplified by PCR using the primers P3 and $\mathrm{P} 4$, primers $\mathrm{P} 5$ and $\mathrm{P} 6$. Then the amplified products were purified and cloned into the pGM-T vector and then sequenced using classical dideoxy Sanger sequencing. Sequencing revealed that the PCR products through primers $\mathrm{P} 3$ and $\mathrm{P} 4$, primers $\mathrm{P} 5$ and P6 corresponded to the predicted length of 3027 and $526 \mathrm{bp}$ in size, respectively, then the sequences were assembled using the Chromas software package to produce the final genome sequence of $3021 \mathrm{bp}$ in length. The isolated virus was identified as DHBV and named SCP01.

Sequence analysis revealed that the genome of DHBV SCP01 (GenBank accession number KM676220) was a double-strand circular DNA and had a size of $3021 \mathrm{bp}$, with a $\mathrm{G}+\mathrm{C}$ content of $43.03 \%$. In addition, the coding region of DHBV SCP01 had three ORFs, designated ORF C, S, and P, which were predicted in the genome by comparison with the proposed structures of other duck hepatitis B viruses nucleotide sequences available in the GenBank of NCBI and identified by the following major criteria: an ATG start codon, a minimum length of $60 \mathrm{bp}$, and less than $60 \%$ overlap with adjacent ORFs, using the online ORF Finder in NCBI. ORFs S, C and $\mathrm{P}$ were predicted to encode the viral surface (preS/S protein: $36.2 \mathrm{kDa}$ and S protein: $18.2 \mathrm{kDa}$ ), the core protein (preC/C protein: $35 \mathrm{kDa}$ and $\mathrm{C}$ protein: $30.3 \mathrm{kDa}$ ), and the polymerase protein ( $\mathrm{P}$ protein: $89.6 \mathrm{kDa}$ ), based on sequence similarities and the presence of conserved domains.

The DHBV SCP01 sequence was aligned with 13 reference strains of DHBV and a Snow goose hepatitis B virus (SGHBV, GenBank accession number AF110998) sequence obtained from GenBank database. The percent nucleotide identity and amino acid identity of DHBV strain SCP01 with those of other avian hepadnaviruses are summarized in Table 2. The DHBV SCP01 genome is the same size as the Western isolates AY250902.1 (Mangisa et al. 2004), AY250904.1 (Mangisa et al. 2004), X12798.1 (Mattes et al. 1990), DQ195079.1 (Sprengel et al. 1985), HQ214130.1, and HQ132730.1. The DHBV SCP01 isolate was $3 \mathrm{nt}$ shorter than the AY494851.1 (Guo et al. 2005), DQ276978.1 and AF110998.1 (SGHBV) (Chang et al. 1999); and 6 nt shorter than the China isolates HM043822.1, AY392760.1, JX469897.1, and M32991.1 (Masako et al. 1989). The differences in length were the result of differences at nt positions $1239-1241$ and 1277-1279 of the 3027 nt genome.
Sequence alignment between the reference strains and the DHBV SCP01 was performed using the Mega 5.1 software, and the neighbor-joining method (Clustal W) was applied. The corresponding phylogenetic tree was constructed using the sequence data of SGHBV, a member of the Avihepadnavirus, as the outgroup. The phylogenetic relationships are presented in Fig. 2. DHBV-XY (GenBank accession number HQ214130.1; origin, Xinyang, China) and DHBV SCP01 formed a branch that was clearly distinct from the other strains. We found that the DHBV SCP01 strain had the highest similarity (99.64 \%) with the sequence of strain DHBV-XY, but had a lower similarity $(90.04 \%)$ with the sequence of strain DHBV CH5 (GenBank accession number EU429325.1; origin, Sichuan, China) isolated from Southwestern China. Comparing the DHBV SCP01 strain with other isolated DHBV strains, the ORF S, C, and P similarities were approximately $88.35-99.90,86.60-99.78$, and 89.62-99.62\%, respectively, and the complete genome sequence similarity was approximately 89.47-99.64\%. This report will aid our understanding of the epidemiology and molecular characteristics of DHBV from Cherry Valley ducks in Southwestern China.

The phylogenetic analysis of the entire nucleotide sequence indicated that the DHBV strain SCP01 was clustered with the strains from Western countries, and it was more closely related to DHBV-XY from central China, than to strains isolated from other areas of China, France, Canada, India, South Africa, Australia, Germany, and the United States. The DHBV strain SCP01 and DHBV-XY belong to "Western" isolates, while others (especially DHBV CH5) belong to "Chinese" isolates. This might be because of the introduction of exotic ducks between countries in recent years during rapid economic development. Phylogenetic analysis of the individual ORFs did not alter the position of the DHBV SCP01 isolate in the trees. Upon translation of ORF P, the DHBV SCP01 isolates were found to share signature amino acids with the Western isolates, as opposed to those of the Chinese isolates (Table 3). The translated sequences of the DHBV SCP01 isolates had a few amino acid changes when compared with the sequences of Western isolates, especially between the DHBV-XY and the DHBV strain SCP01, which were mostly in the polymerase protein (three amino acid changes).

\section{Conclusion}

This study show that the DHBV SCP01 strain from Sichuan belonged to "Western" isolates, while the DHBV CH5 from Sichuan belonged to "Chinese" isolates. The complete molecular characterization of the DHBV SCP01 strain will contribute to further studies on molecular epidemiology and enable the 
Table 2 Similarities analysis with the 15 isolates of avian hepadnavirus

\begin{tabular}{|c|c|c|c|c|c|c|}
\hline Number & GenBank & Complete genome $^{a}$ & Polymerase protein ${ }^{b}$ & Genomic length (nt) & Avain species & Location \\
\hline 1 & AY250902.1 & 99.47 & 99.49 & 3021 & Pekin duck & South Africa \\
\hline 2 & AY250904.1 & 99.37 & 99.24 & 3021 & Pekin duck & South Africa \\
\hline 3 & X12798.1 & 98.58 & 98.09 & 3021 & Duck & Germany \\
\hline 4 & DQ195079.1 & 94.24 & 91.09 & 3021 & Pekin duck & Germany \\
\hline 5 & AY494851.1 & 91.39 & 88.04 & 3024 & Puna teal & USA \\
\hline 6 & HQ214130.1 & 99.64 & 99.62 & 3021 & Cherry valley duck & Henan \\
\hline 7 & HQ132730.1 & 94.47 & 91.60 & 3021 & Duck & Guangdong \\
\hline 8 & DQ276978.1 & 90.40 & 91.48 & 3024 & Tadorna tadorna & Hubei \\
\hline 9 & HM043822.1 & 90.07 & 91.35 & 3027 & Cherry valley duck & Henan \\
\hline 10 & EU429325.1 & 90.04 & 87.91 & 3006 & Anas platyrhynchos & Sichuan \\
\hline 11 & AY392760.1 & 90.93 & 86.64 & 3027 & Brown duck & Guangdong \\
\hline 12 & JX469897.1 & 90.07 & 86.51 & 3027 & Mallard & Guilin \\
\hline 13 & M32991.1 & 89.47 & 84.61 & 3027 & White Shanghai duck & Shanghai \\
\hline 14 & AF110998.1 & 89.14 & 85.37 & 3024 & Anser caerulescens & Germany \\
\hline 15 & KM676220.1 & & & 3021 & Cherry valley duck & Sichuan \\
\hline
\end{tabular}

Boldface indicates the highest, and italic, the lower

a Percent nucleotide identity

b Percent amino acid identity

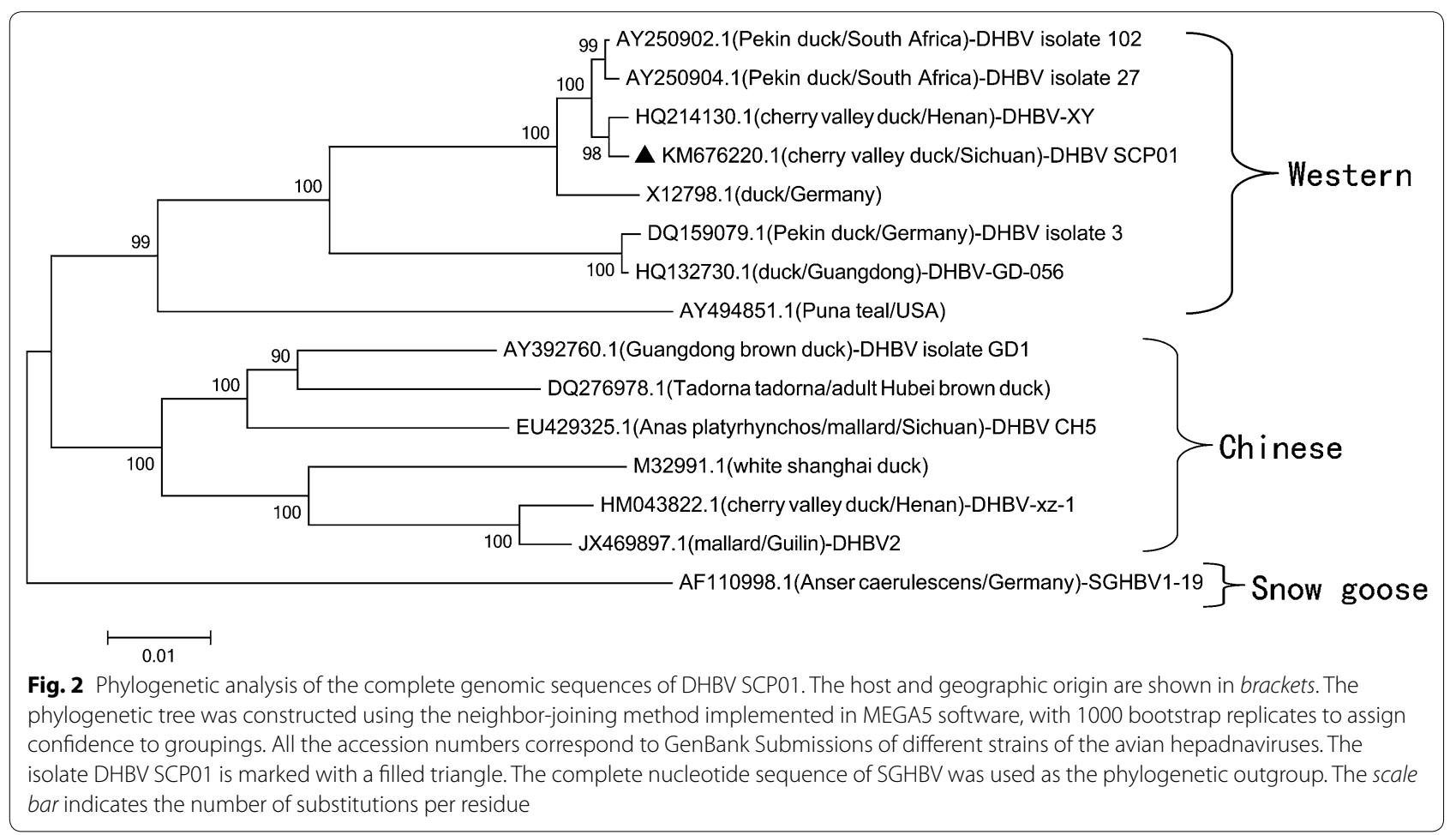

development of better measures to control DHBV. To date, there are no effective, preventive vaccines against DHBV in poultry, and the nature of circulating DHBV and HBV have remained largely elusive in China. Our purpose in submitting this report is our hope that these data will promote investigations by others in the virology community into the evolutionary biology, epidemiology and pathobiology of hepadnavirus infections. In 
Table 3 Comparison of amino acid residues of DHBV SCP01 isolates with those of other avain hepadnaviruses

\begin{tabular}{|c|c|c|c|c|c|c|c|}
\hline ORF region & $\begin{array}{l}\text { Signature amino } \\
\text { acid residue }^{\mathrm{a}}\end{array}$ & $\begin{array}{l}\text { HQ214130 } \\
\text { DHBV-XY }\end{array}$ & $\begin{array}{l}\text { KM676220 } \\
\text { DHBV SCP01 }\end{array}$ & Western isolates & Chinese isolates & $\begin{array}{l}\text { EU429325.1DHBV } \\
\text { CH5 }\end{array}$ & Exceptions $^{b}$ \\
\hline \multirow{16}{*}{$\begin{array}{l}\text { Polymerase } \\
\text { Spacer }\end{array}$} & 202 & $\mathrm{H}$ & $\mathrm{H}$ & $\mathrm{H}$ & $Y$ & $Y$ & \\
\hline & 208 & Q & $\mathrm{H}$ & $H$ & Q & Q & \\
\hline & 210 & Y & Y & Y & $P$ & $P$ & \\
\hline & 223 & $\mathrm{~T}$ & $\mathrm{~T}$ & $\mathrm{~T}$ & $E$ & $E$ & \\
\hline & 231 & V & V & V & A & A & \\
\hline & 237 & K & K & K & $P$ & $P$ & \\
\hline & 244 & $\mathrm{~F}$ & $\mathrm{~F}$ & $\mathrm{~F}$ & C & $\mathrm{S}$ & \\
\hline & 272 & $S$ & S & $S$ & $\mathrm{~T}^{\mathrm{b}}$ & $S$ & X58569(P) \\
\hline & 345 & $S$ & S & $S$ & Q & Q & \\
\hline & 356 & V & V & V & $E$ & $E$ & \\
\hline & 360 & G & G & G & $\mathrm{R}$ & $\mathrm{R}$ & \\
\hline & 566 & $N$ & $N$ & $N$ & $K^{b}$ & K & $\times 58568 / 9(R)$ \\
\hline & 635 & S & S & $\mathrm{S}$ & $A$ & $A$ & \\
\hline & 694 & V & V & V & 1 & 1 & \\
\hline & 700 & $S$ & $\mathrm{~S}$ & $S$ & V & V & \\
\hline & 769 & $P$ & $P$ & $P$ & $\mathrm{~T}$ & $\mathrm{~T}$ & \\
\hline
\end{tabular}

a Numbering of amino acid residues is according to 3021 base pair complete genome

b Exceptions: the alternative amino acid residue is shown in the square brackets

addition, continuing duck hepatitis B virus surveillance in poultry is critical to understanding DHBV infection, and to find further animal infection models to study HBV infection.

\section{Abbreviations}

DHBV: duck hepatitis B virus; HBV: hepatitis B virus; SGHBV: snow goose hepatitis B virus; HCC: hepatocellular carcinoma; NCBI: National Center for Biotechnology Information; ORFs: open reading frames; DR: direct repeat.

\section{Authors' contributions}

QL designed the study, performed the experiments, participated in the sequence alignment and wrote the manuscript. RJ, AC, MW, DZ, ML and SC critically revised the experiment design and manuscript. ZY, BJ and SL participated in the design of the study and helped to revise the manuscript. All authors read and approved the final manuscript. QL is considered as first author

\section{Author details}

1 Avian Disease Research Center, Sichuan Agricultural University, Chengdu 611130, China. ${ }^{2}$ Institute of Preventive Veterinary Medicine, Sichuan Agricultural University, Chengdu 611130, China. ${ }^{3}$ Key Laboratory of Animal Disease and Human Health of Sichuan Province, Chengdu 611130, China.

\section{Acknowledgements}

This work was supported by National Science and Technology Support Program (2015BAD12B05), China Agricultural Research System (CARS-43-8), Integration and Demonstration of Key Technologies for Duck Industrial in Sichuan Province (2014NZ0030), Sichuan Province Research Programs (2013HH0042/2 013TD0015/2014-002)

\section{Competing interests}

The authors declare that they have no competing interests.

\section{Compliance with ethical standards}

All procedures followed for the use of animals in these studies were in accordance with the ethical standards and was approved by the Institutional Animal
Care and Use Committee. This article does not contain any studies with human participants performed by any of the authors. All authors read and approved the final manuscript.

Received: 27 January 2016 Accepted: 2 August 2016

Published online: 17 August 2016

\section{References}

Chang S-F, Netter HJ, Bruns M, Schneider R, Frölich K, Will H (1999) A new avian hepadnavirus infecting snow geese (Anser caerulescens) produces a significant fraction of virions containing single-stranded DNA. Virology 262(1):39-54

Cova L, Abdul F, Buronfosse T (2011) Avihepadnavirus. The springer index of viruses, pp 615-624

Feng F, Teoh CQ, Qiao Q, Boyle D, Jilbert AR (2010) The development of persistent duck hepatitis B virus infection can be prevented using antiviral therapy combined with DNA or recombinant fowlpoxvirus vaccines. Vaccine 28(46):7436-7443

Günther S, Li B-C, Miska S, Krüger D, Meisel H, Will H (1995) A novel method for efficient amplification of whole hepatitis $B$ virus genomes permits rapid functional analysis and reveals deletion mutants in immunosuppressed patients. J Virol 69(9):5437-5444

Guo H, Mason WS, Aldrich CE, Saputelli JR, Miller DS, Jilbert AR, Newbold JE (2005) Identification and characterization of avihepadnaviruses isolated from exotic anseriformes maintained in captivity. J Virol 79(5):2729-2742

Jilbert A, Kotlarski I (2000) Immune responses to duck hepatitis B virus infection. Dev Comp Immunol 24(2):285-302

Lavanchy D, Kane M (2016) Global epidemiology of hepatitis B virus infection. In: Hepatitis B virus in human diseases. Springer, New York, pp 187-203

Liu Q, Jia R, Wang M, Huang J, Zhu D, Chen S, Yin Z, Wang Y, Chen X, Cheng A (2014) Cloning, expression and purification of duck hepatitis B virus (DHBV) core protein and its use in the development of an indirect ELISA for serologic detection of DHBV infection. Arch Virol 159(5):897-904 
Mangisa NP, Smuts HE, Kramvis A, Linley CW, Skelton M, Tucker TJ, Hall PDLM, Kahn D, Jilbert AR, Kew MC (2004) Molecular characterization of duck hepatitis B virus isolates from South African ducks. Virus Genes 28(2):179-186

Masako U, Esumi M, Shikata T (1989) Molecular cloning and sequence analysis of duck hepatitis B virus genomes of a new variant isolated from Shanghai ducks. Virology 173(2):600-606

Mason W, Seal G, Summers J (1980) Virus of Pekin ducks with structural and biological relatedness to human hepatitis B virus. J Virol 36(3):829-836

Mattes F, Tong S, Teubner K, Blum H (1990) Complete nucleotide sequence of a German duck hepatitis B virus. Nucleic Acids Res 18(20):6140

Molnar-Kimber KL, Summers JW, Mason WS (1984) Mapping of the cohesive overlap of duck hepatitis B virus DNA and of the site of initiation of reverse transcription. J Virol 51(1):181-191
Sprengel R, Kuhn C, Will H, Schaller H (1985) Comparative sequence analysis of duck and human hepatitis B virus genomes. J Med Virol 15(4):323-333

Tamura K, Peterson D, Peterson N, Stecher G, Nei M, Kumar S (2011) MEGA5: molecular evolutionary genetics analysis using maximum likelihood, evolutionary distance, and maximum parsimony methods. Mol Biol Evol 28(10):2731-2739

Triyatni M, Ey PL, Tran T, Le Mire M, Qiao M, Burrell CJ, Jilbert AR (2001)

Sequence comparison of an Australian duck hepatitis B virus strain with other avian hepadnaviruses. J Gen Virol 82(2):373-378

\section{Submit your manuscript to a SpringerOpen ${ }^{\circ}$ journal and benefit from:}

- Convenient online submission

- Rigorous peer review

- Immediate publication on acceptance

- Open access: articles freely available online

- High visibility within the field

- Retaining the copyright to your article 